Gazi University
Journal of Science
http://dergipark.gov.tr/gujs

\title{
Synthesis of New Ligands Containing Azomethine Group and Investigation of Antioxidant, Antiurease Activities
}

\author{
Nurhan GUMRUKCUOGLU ${ }^{1, *}$ (i) , Bahar BILGIN SOKMEN ${ }^{2}$ (D) \\ ${ }^{I}$ Department of Chemistry, Faculty of Science, Karadeniz Technical University, 61080 Trabzon, Turkey \\ ${ }^{2}$ Department of Chemistry, Faculty of Arts and Sciences, Giresun University, 28049, Giresun, Turkey
}

\author{
Highlights \\ - As standard antioxidant, Trolox (6-hydroxy-2,5,7,8-tetramethylchroman-2-carboxylic acid) was used. \\ - The reducing power activities of the compounds were compared with BHT. \\ - Values were the means of three replicates \pm Standard deviation
}

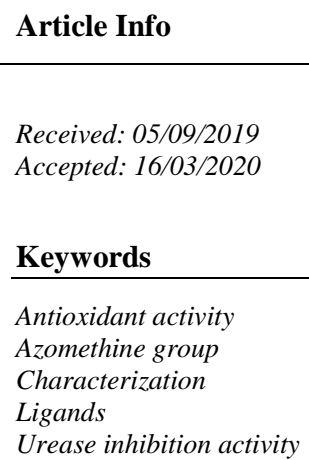

\section{INTRODUCTION}

Schiff bases are used for obtaining thermotropic liquid crystallized polymers as salat ligands in coordination chemistry, as a dioxygen carrier for metal treatment as radiopharmacologies, modeling systems for biological macromolecules and in catalytic reactions [1-3]. Also, Schiff bases containing phenyl in aldehyde or amine structures in acidic ambiance are corrosion inhibiting for aluminum [4-6]. The geometrical structures of Schiff bases can give us an idea about the complexity of the complexes. The complexes obtained with Schiff bases having a more rigid structure are more likely to form a polymeric structure [7].

It has been reported that structurally modified derivatives of Schiff bases can be used in the pharmaceutical industry as a bioactive substance in drugs with metal coordinated complexes. Bonding with metals thanks to nitrogen and sulfur donor atoms due to its antitumor, antibacterial, antimicrobial and antifungal properties and their effects on the reproductive system of the thiosemicarbazones potentially present in the presence of nitrogen and sulfur donor atoms, the heterocyclic complexes obtained by B (III), Pd (II), and $\mathrm{Pt}$ (II). The researches on these issues are widely included in the literature [8-11].

Studies on Schiff bases that can reduce the effects of radiation have been made. Researchers have found that azobenzene Schiff bases can absorb UV radiation with cis-trans change; thus, they suggested that the harmful effect of radiation could be transformed [12]. Surgical procedures and chemotherapy drugs are currently used in the treatment of cancer cells. These drugs have many side effects as well as healing effects. 
Studies on new metal-based anticancer drugs to reduce these side effects of drugs continue rapidly. Also, it has been observed that all polymer-metal complexes have high thermal stability [13, 14].

Oxidative agents play a significant role in plays an important role in various diseases, such as carcinogenesis, atherosclerosis, diabetes, nefritisis, cardiovascular diseases and neurodegenerative diseases [15-17]. For these reason, new synthetic antioxidants are requested from exterior sources [18]. The urease (urea amidohydrolase EC 3.5.1.5) is a nickel-dependent enzyme which catalyzes the hydrolysis of urea to carbon dioxide and ammonia. Ureases are widely found in various plants, algae, fungi and bacteria [19, 20]. In human, excess urease activity can be harmful to health like kidney stones, peptic and duodenal ulcers, urinary catheter encrustation, hepatic coma, gastric cancers and pyelonephritis [21]. Urease is well known for the pathologies caused by Gram (-) bacterium Helicobacter pylori which is play a crucial role in the pathogenesis of especially ulcer and cancer (gastric and peptic) [22]. In the past decades, various urease inhibitors have been studied such as phosphorodiamidates, boric acid, humic acid, citric acid, hydroxamic acid etc. $[23,24]$. As a result, in this research urease inhibitors with good activity are expected.

Keeping in view the importance of Triazol, Shiff's bases, and reduction products our aimed is to the develop hybrid molecules through the combination of different pharmacophores in one frame to utilize as ligands and to study the ligands and their metal complexes from a structural point of view. Metal chelates of Schiff bases hold exciting possibilities for the future concerning their wide applications viz. in designing new catalytic systems, in formulating new synthetic route, in developing new analytical reagents and in metalbased antimicrobial agents etc., In addition, the synthesis of a compound can be used in a selective extraction of the metal is of great importance for the environment and the metal industry.

In current research, synthesized new ligands containing Azomethine group with high efficiency. Antioxidant and antiurease activities of new ligands were determined by in vitro assay and compared to the activity of standard compounds.

\section{EXPERIMENTAL}

\subsection{General}

All synthesized compounds were sensitive to air humidity and oxygen, so all experiments were carried out in an inert atmosphere and Schlenk technique was used in the reactions. Glass materials used in reactions before use, a vacuum was applied and heated to remove the moisture and oxygen contained and then filled with argon gas. Ethyl p-methylbenzimidate hydrochloride 1 was synthesized benefiting a published method [25]. Antioxidant activities of compounds were measured spectrophotometrically (UV-1240, Shimadzu, Japan).

\subsection{Ethyl N'-Furan-2-Carbonyl-p-methylbenzohydrazonate (2)}

To the solution of ethyl p-methyl benzimidate hydrochloride $1(0.01 \mathrm{~mol})$ in dry ethanol and the solution of furan-2-carboxylic acid hydrazide $(0.01 \mathrm{~mol})$ in dry ethanol were mixtured and stirred at $0{ }^{\circ} \mathrm{C}$ for $6 \mathrm{~h}$. Then, ammonium chloride was separated by filtration. Finally a white solid matter was obtained, purified by ethylacetate to determined compound 2. "Yield, $1.74 \mathrm{~g}(64.00 \%)$ "; " white powder, $\mathrm{mp} 162-163{ }^{\circ} \mathrm{C}$ (EtOH)"; IR ( $\left.\mathrm{cm}^{-1}\right)$ : [699-771, 851 (mono, 1, 4 di substitute arom. ring); $1616(v \mathrm{C}=\mathrm{N}) ; 1674(v \mathrm{C}=\mathrm{O})$; $3316(v \mathrm{NH})$ ]; ${ }^{1} \mathrm{H}$ NMR $(\mathrm{J}, \mathrm{Hz}): 1.32\left(3 \mathrm{H}, \mathrm{t}, \mathrm{J}=6.90, \mathrm{CH}_{2} \mathrm{CH}_{3}\right) ; 2.38\left(3 \mathrm{H}, \mathrm{s}, \mathrm{Ar}_{-} \mathrm{CH}_{3}\right) ; 4.26(2 \mathrm{H}, \mathrm{q}, \mathrm{J}=6.90$, $\left.\mathrm{CH}_{2} \mathrm{CH}_{3}\right)$; Ar-H [7.30 (2H, d, H-1, J=7.80, p-tolyl); 7.95 (2H, d, H-2, J=7.80, p-tolyl); 8.08-8.71 (3H, m, $\mathrm{H}-3,4,5$, furyl)]; 10.76 ( $1 \mathrm{H}, \mathrm{s}, \mathrm{NH}) ;{ }^{13} \mathrm{C} \mathrm{NMR}(\delta, \mathrm{ppm}): 10.04\left(\mathrm{CH}_{3}\right) ; 21.16\left(\mathrm{Ar}-\mathrm{CH}_{3}\right) ; 63.01\left(\mathrm{OCH}_{2}\right) ; \mathrm{Ar}-$ C [124.72 (C); $131.65(2 \mathrm{CH}) ; 132.13(2 \mathrm{CH}) ; 133.02(\mathrm{CH}) ; 134.19(\mathrm{C}) ; 139.26(\mathrm{CH}) ; 145.90(\mathrm{C}) ; 150.32$ $(\mathrm{CH})] ; 167.18(\mathrm{C}=\mathrm{N}) ; 170.04(\mathrm{C}=\mathrm{O})$; Anal.calcd. Found, \%: C 66.21; H 5.87; N 10.26. $\mathrm{C}_{15} \mathrm{H}_{16} \mathrm{~N}_{2} \mathrm{O}_{3}$. Calculated, \%: C 66.17; H 5.92; N 10.29. 
A hydrazine hydrate solution $(0.01 \mathrm{~mol})$ and compound $2(0.005 \mathrm{~mol})$ were dissolved in 1-hydroxy propan $(50 \mathrm{~mL})$, after the mixture was refluxed for $18 \mathrm{~h}$, left to cool. Then removed from the precipitate formed. The product was purified with ethylacetate to obtained compound 3. "Yield, $2.02 \mathrm{~g}(84.13 \%)$ ); "white powder, mp 183-184 ${ }^{\circ} \mathrm{C}\left(\mathrm{CH}_{3} \mathrm{COOEt}\right)$ "; IR $\left(\mathrm{cm}^{-1}\right)$ : [706-770, 838 (mono, 1,4 di substitue arom. ring); $\left.1615,1617(v 2 \mathrm{C}=\mathrm{N}) ; 3194-3321\left(v \mathrm{NH}_{2}\right)\right] ;{ }^{1} \mathrm{H} \mathrm{NMR}(\mathrm{J}, \mathrm{Hz}): 2.39\left(3 \mathrm{H}, \mathrm{s}, \mathrm{Ar}-\mathrm{CH}_{3}\right), 6.28\left(2 \mathrm{H}, \mathrm{s}, \mathrm{NH}_{2}\right), \mathrm{Ar}-$ H [7.56 (2H, d, H-1, J=7.80, p-tolyl), 7.89 (2H, d, H-2, J=7.80 p-tolyl), 8.05-8.75 (3H, m, H-3,4,5, furyl)]; ${ }^{13} \mathrm{C}$ NMR $(\delta \mathrm{ppm}): 20.82\left(\mathrm{Ar}-\mathrm{CH}_{3}\right) ; \mathrm{Ar}-\mathrm{C}[123.88(\mathrm{CH}) ; 124.90(\mathrm{C}, \mathrm{Ph}) ; 127.42(2 \mathrm{CH}, \mathrm{Ph}) ; 128.15(2 \mathrm{CH}$, $\mathrm{Ph}) ; 134.56(\mathrm{C}, \mathrm{Ph}) ; 138.90(\mathrm{CH}) ; 147.01(\mathrm{C}) ; 149.98(\mathrm{CH})]$; $153.11,155.00\left(2 \mathrm{C}\right.$, triazole $\left.\mathrm{C}_{5}, \mathrm{C}_{3}\right)$; Anal.calcd. Found, \%: C 64.98; H 5.05; N 23.29. $\mathrm{C}_{13} \mathrm{H}_{12} \mathrm{~N}_{4} \mathrm{O}$. Calculated, \%: C 64.99; H 5.03; N 23.32.

\subsection{Synthesis of Arylidenamino Compounds $(4 a-c)$}

The aldehyde compound $(1 \mathrm{mmol})$ was dissolved completely in ethyl alcohol $(30 \mathrm{~mL})$ and the amine compound (1 mmol) was added. Half of alcohol was removed in vacuum after boiling for 4 hours in inert atmosphere and crystallized by addition of diethylether. The resulting crystals were cleaned with diethylether and dried in vacuo.

\subsubsection{3-Furan-5-(p-methylphenyl-4-yl)-4-(2-Hydroxy-1-benzylidenamino)-4H-1,2,4-Triazole (4a)}

"Yield, 2.80 g (81.44 \%)"; "white powder, mp 148-149 ${ }^{\circ} \mathrm{C}(\mathrm{EtOH})$ "; IR $\left(\mathrm{cm}^{-1}\right)$ : [695-768, 743, 819 (mono, 1,2, 1,4 di substitue) arom. ring], 1530, $1595(v 2 \mathrm{C}=\mathrm{N}), 3309(v \mathrm{OH}) ; 1 \mathrm{H}$ NMR (J, Hz): $2.34(3 \mathrm{H}, \mathrm{s}, \mathrm{Ar}-$ $\left.\mathrm{CH}_{3}\right), \mathrm{Ar}-\mathrm{H}[7.30(2 \mathrm{H}, \mathrm{d}, \mathrm{H}-1, \mathrm{~J}=7.80$, p-tolyl $), 7.60(1 \mathrm{H}, \mathrm{t}, \mathrm{H}-8, \mathrm{~J}=7.02,2$-hydroxyphenyl), 7.71 (2H, d, H-2, J=7.80, p-tolyl), 7.75 (1H, t, H-7, J=7.02, 2-hydroxyphenyl), 7.80-8.07 (4H, m, H-6, 9, 10, 11, 2hydroxyphenyl), 8.10-8.51 (3H, m, H-3,4,5, furyl)]; $8.69(1 \mathrm{H}, \mathrm{s}, \mathrm{N}=\mathrm{CH}), 10.30(1 \mathrm{H}, \mathrm{s}, \mathrm{OH}) ;{ }^{13} \mathrm{C} \mathrm{NMR}(\delta$, ppm): $21.11\left(\mathrm{Ar}_{-} \mathrm{CH}_{3}\right) ; \mathrm{Ar}-\mathrm{C}[120.08(\mathrm{CH}) ; 121.23 ;(2 \mathrm{CH}, \mathrm{Ph}) ; 122.54(\mathrm{CH}) ; 123.36(\mathrm{CH}), 125.34(\mathrm{CH})$; 127.45 (2CH); $129.13(\mathrm{CH}) ; 129.98(\mathrm{C}) ; 130.12(\mathrm{CH}) ; 135.80(\mathrm{C}) ; 137.40(\mathrm{C}) ; 138.67$ (C); $139.23(\mathrm{C})$; $149.10(\mathrm{CH}) ; 150.12,152.75\left(2 \mathrm{C}\right.$, triazole $\left.\mathrm{C}_{5}, \mathrm{C}_{3}\right) ; 171.52(\mathrm{~N}=\mathrm{CH})$; Anal.calcd. Found, \%: $\mathrm{C} 69.73 ; \mathrm{H}$ 4.66; $\mathrm{N}$ 16.29. $\mathrm{C}_{20} \mathrm{H}_{16} \mathrm{~N}_{4} \mathrm{O}_{2}$. Calculated, \%: C 69.76; $\mathrm{H} 4.68 ; \mathrm{N} 16.27$.

\subsubsection{3-Furan-5-(p-methylphenyl-4-yl)-4-(1-Naphthylidenamino)-4H-1,2,4-Triazole (4b)}

"Yield, 3.03 g (80.16\%)"; "white powder, mp180-181 ${ }^{\circ} \mathrm{C}(\mathrm{EtOH})$ "; IR $\left(\mathrm{cm}^{-1}\right)$ : [690-728, 699-773, 813 (2 mono, 1,4 di substitue) arom. ring], 1593, 1629 ( v 2C=N); ${ }^{1} \mathrm{H}$ NMR (J, Hz): $2.33\left(3 \mathrm{H}, \mathrm{s}, \mathrm{Ar}-\mathrm{CH}_{3}\right), \mathrm{Ar}-\mathrm{H}$ [6.90-7.04 (3H, m, H-3,4,5, furyl), 7.06-7.18 (m, 1H, H-8, 1-naphthyl), 7.36 (2H, d, H-1, J=7.80, p-tolyl), 7.48-7-60 (2H, m, H-7, 11, 1-naphthyl), 7.86-8.00 (2H, m, H-2, p-tolyl), 8.06 (1H, d, H-12, J=8.76, 1naphthyl), 8.34 (1H, d, H-6, J=8.76, 1-naphthyl), 8.60-8.89 (2H, m, H-9, 10, 1-naphthyl)]; 9.20 (1H, s, $\mathrm{N}=\mathrm{CH}) ;{ }^{13} \mathrm{C}$ NMR $(\delta, \mathrm{ppm}): 20.22\left(\mathrm{Ar}_{-} \mathrm{CH}_{3}\right) ; \mathrm{Ar}-\mathrm{C}[109.43(\mathrm{C}) ; 116.11(\mathrm{CH}) ; 122.04(2 \mathrm{CH}) ; 123.00(\mathrm{C})$; $123.16(\mathrm{CH}) ; 123.75(\mathrm{CH}) ; 124.07(\mathrm{C}) ; 125.04(\mathrm{CH}) ; 126.55(\mathrm{CH}) ; 127.66(2 \mathrm{CH}) ; 129.87(\mathrm{CH}) ; 133.12$ $(\mathrm{CH}) ; 135.89(\mathrm{CH}) ; 137.12(\mathrm{CH}), 138.77(\mathrm{C}) ; 148.70(\mathrm{C}) ; 149.24(\mathrm{CH}) ; 150.87(\mathrm{CH}) ; 160.99(\mathrm{C})] ; 151.24$, 153.76, $\left(2 \mathrm{C}\right.$, triazole $\left.\mathrm{C}_{5}, \mathrm{C}_{3}\right), 167.02(\mathrm{~N}=\mathrm{CH})$; Anal.calcd. Found, \%: C 76.15; H 4.78; N 14.84. $\mathrm{C}_{24} \mathrm{H}_{18} \mathrm{~N}_{4} \mathrm{O}$. Calculated, \%: C 76.18; H 4.79; N 14.80.

\subsubsection{3-Furan-5-(p-methylphenyl-4-yl)-4-(2-Hydroxy-1-Naphthylidenamino)-4H-1,2,4-Triazole (4c)}

"Yield, 3.22 g (81.78 \%)"; "white powder, mp191-192 ${ }^{\circ} \mathrm{C}(\mathrm{EtOH})$ "; IR $\left(\mathrm{cm}^{-1}\right)$ : [695-771, 747, 832 (mono, 1,2, 1,4 di substitue) arom. ring]; 1626, $1640(v 2 \mathrm{C}=\mathrm{N}) ; 3318(v \mathrm{OH}) ; 1 \mathrm{H}$ NMR (J, Hz): $2.33(3 \mathrm{H}, \mathrm{s}, \mathrm{Ar}-$ $\left.\mathrm{CH}_{3}\right) ; \mathrm{Ar}-\mathrm{H}[7.25$ (2H, d, H-1, J=7.80, p-tolyl); 7.39 (2H, d, H-2, J=7.80, p-tolyl); 7.44 (1H, t, J=8.65, H8, 2-hydroxy-1-naphthyl); 7.56-7.69 (2H, m, H-6, 9, 2-hydroxy-1-naphthyl); 7.73 (1H, t, J=8.65, H-7, 2hydroxy-1-naphthyl); 7.75-8.05 (2H, m, H-10, 11, 2-hydroxy-1-naphthyl); $9.22(1 \mathrm{H}, \mathrm{s}, \mathrm{N}=\mathrm{CH}) ; 10.04(1 \mathrm{H}$, s, $\mathrm{OH}) ;{ }^{13} \mathrm{C}$ NMR $(\delta, \mathrm{ppm}): 20.45\left(\mathrm{Ar}-\mathrm{CH}_{3}\right) ; \mathrm{Ar}-\mathrm{C}[113.24(\mathrm{C}) ; 117.36(\mathrm{CH}) ; 119.14(\mathrm{C}) ; 121.33(2 \mathrm{CH})$; $123.00(\mathrm{C}) ; 124.35(\mathrm{CH}) ; 124.55(\mathrm{CH}) ; 124.91(\mathrm{CH}) ; 127.88(2 \mathrm{CH}) ; 128.09(\mathrm{CH}) ; 128.46(\mathrm{CH}) ; 130.05$ (C); $134.58(\mathrm{C}) ; 136.12(\mathrm{CH}) ; 138.09(\mathrm{CH}) ; 142.55(\mathrm{C}) ; 150.02(\mathrm{CH}) ; 160.13(\mathrm{C})] ; 149.77,150.54(2 \mathrm{C}$, triazole $\left.\mathrm{C}_{5}, \mathrm{C}_{3}\right) ; 164.56(\mathrm{~N}=\mathrm{CH})$; Anal. calcd. Found, \%: $\mathrm{C} 73.03 ; \mathrm{H} 4.65 ; \mathrm{N}$ 14.22. $\mathrm{C}_{24} \mathrm{H}_{18} \mathrm{~N}_{4} \mathrm{O}_{2}$. Calculated, $\%$ : C 73.09; H 4.60; N 14.20. 


\subsection{Synthesis of Arylamino Ligands (5a-c)}

The arilidenamino ligands $(4 \mathrm{a}-\mathrm{c})(0.005 \mathrm{~mol})$ and pured methanol $(30 \mathrm{~mL})$ were mixed, and sodium borohydride $(0.01 \mathrm{~mol})$ was attended carefully to the solution. The solution was boiled for $25 \mathrm{~min}$ and then left to warm. After being evapora, the resulting solid product was washed with water for three times. After the moisture of the substance is dried, the matter was purified with a suitable solvent.

$\mathrm{S}$

\subsubsection{3-Furan-5-(p-methylphenyl-4-yl)-4(2-Hydroxy-1-benzylamino)-4H-1,2,4-Triazole (5a)}

"Yield, $3.01 \mathrm{~g}$ (87.00 \%)"; "white powder, mp 176-177 ${ }^{\circ} \mathrm{C}(\mathrm{EtOH})$ "; IR $\left(\mathrm{cm}^{-1}\right)$ : [746, 691-774, 857 (1,2 di, mono, 1,4 di substitue) arom. ring]; 1568, $1612(v 2 \mathrm{C}=\mathrm{N}) ; 3278(v \mathrm{NH}) ; 3304(v \mathrm{OH}) ;{ }^{1} \mathrm{H} \mathrm{NMR}(\mathrm{J}, \mathrm{Hz})$ : $2.39\left(3 \mathrm{H}, \mathrm{s}, \mathrm{Ar}-\mathrm{CH}_{3}\right) ; 3.92\left(2 \mathrm{H}, \mathrm{d}, \mathrm{J}=4.56,-\mathrm{NHCH}_{2}\right) ; 6.74\left(1 \mathrm{H}, \mathrm{t}, \mathrm{J}=4.56,-\mathrm{NHCH}_{2}\right) ; \mathrm{Ar}-\mathrm{H}[6.95(2 \mathrm{H}$, m, H-2, p-tolyl); 7.17 (2H, d, H-1, J=7.80, p-tolyl); 7.25 (2H, m, H-7, 8, 2-hydroxyphenyl); 7.48-7.55 (3H, $\mathrm{m}$, furyl); 7.87-7.89 (2H, m, H-6, 9, 2-hydroxyphenyl)]; 10.36 (s, $1 \mathrm{H}, \mathrm{OH}) ;{ }^{13} \mathrm{C} \mathrm{NMR}(\delta$, ppm): $20.96(\mathrm{Ar}-$ $\left.\mathrm{CH}_{3}\right) ; 52.47\left(\mathrm{CH}_{2}\right) ; \mathrm{Ar}-\mathrm{C}[120.31(\mathrm{CH}) ; 121.66(\mathrm{CH}) ; 122.99(2 \mathrm{CH}) ; 125.89(\mathrm{CH}) ; 126.54(2 \mathrm{CH}) ; 127.23$ (C); $129.76(\mathrm{C}) ; 130.32(\mathrm{C}) ; 138.67(\mathrm{CH}) ; 142.56(\mathrm{CH}) ; 143.90(\mathrm{C}) ; 144.26(\mathrm{CH}) ; 151.06(\mathrm{C}) ; 152.12$ $(\mathrm{CH})] ; 150.45,151.18\left(2 \mathrm{C}\right.$, triazole $\left.\mathrm{C}_{5}, \mathrm{C}_{3}\right)$; Anal.calcd. Found, \%: C 69.34; H 5.21; N 16.22. $\mathrm{C}_{20} \mathrm{H}_{18} \mathrm{~N}_{4} \mathrm{O}_{2}$. Calculated, \%: C 69.35; H 5.24; N 16.17.

\subsubsection{3-Furan-5-(p-methylphenyl-4-yl)-4-(1- Triazole (5b): Naphthylamino)-4H-1,2,4-Triazole (5b)}

"Yield, 3.14 g (82.78 \%)"; "white powder, mp 184-185 ${ }^{\circ} \mathrm{C}(\mathrm{EtOH})$ "; IR $\left(\mathrm{cm}^{-1}\right)$ : [692-771, 698-763, 854, (2 mono, 1,4 di substitue) arom. ring], 1535, $1626(v 2 \mathrm{C}=\mathrm{N}), 3213(v \mathrm{NH}) ;{ }^{1} \mathrm{H}$ NMR (J, Hz): $2.41(3 \mathrm{H}, \mathrm{s}$, Ar- $\left.\mathrm{CH}_{3}\right) ; 4.29\left(2 \mathrm{H}, \mathrm{d},-\mathrm{NHCH}_{2}, \mathrm{~J}=4.56\right) ; 6.80\left(1 \mathrm{H}, \mathrm{t}, \mathrm{NHCH}_{2}, \mathrm{~J}=4.56\right) ; \mathrm{Ar}-\mathrm{H}$ [7.14 (2H, d, J=7.80, p-tolyl); 7.32 (2H, t, H-8, 11, J=8.76, 1-naphthyl); 7.41-7.60 (3H, m, furyl); 7.77 (2H, d, J=7.80, p-tolyl); 7.81-7.95 (2H, m, H-10, 12, 1-naphthyl); 8.00-8.35 (3H, m, H-6, 7, 9, 1-naphthyl)]; ${ }^{13} \mathrm{C}$ NMR $(\delta$, ppm): 20.96 (Ar$\left.\mathrm{CH}_{3}\right)$; $46.88\left(\mathrm{CH}_{2}\right)$; $\mathrm{Ar}-\mathrm{C}[112.55(\mathrm{C}) ; 118.32(\mathrm{C}) ; 122.12(2 \mathrm{CH}) ; 123.17(\mathrm{CH}) ; 123.66(\mathrm{C}) ; 126.30(\mathrm{C})$; $127.04(2 \mathrm{CH}) ; 127.85(\mathrm{CH}) ; 128.66(\mathrm{CH}) ; 129.40(\mathrm{CH}) ; 129.23(\mathrm{CH}) ; 133.09(\mathrm{CH}) ; 137.91(\mathrm{CH}) ; 138.32$ (C); $138.70(\mathrm{CH}) ; 149.01(\mathrm{C}) ; 149.18(\mathrm{CH}) ; 149.76(\mathrm{CH}) ; 156.42(\mathrm{C})] ; 152.50,154.89\left(2 \mathrm{C}\right.$, triazole $\mathrm{C}_{5}$, $\mathrm{C}_{3}$ ); Anal.calcd. Found, \%: C 75.71; H 5.34; N 14.76. $\mathrm{C}_{24} \mathrm{H}_{20} \mathrm{~N}_{4} \mathrm{O}$. Calculated, \%: C 75.77; H 5.30; N 14.73.

\subsubsection{3-Furan-5-(p-methylphenyl-4-yl)-4-(2-Hydroxy-1-Naphthylamino)-4H-1,2,4-Triazole (5c)}

"Yield, 3.46 g (87.41 \%)"; "white powder, mp 191-192 ${ }^{\circ} \mathrm{C}(\mathrm{EtOH})$ "; IR $\left(\mathrm{cm}^{-1}\right)$ : [725-707, 738, 810 (mono, 1,2, 1,4 di substitue) arom. ring]; 1588, $1619(v 2 \mathrm{C}=\mathrm{N}) ; 3293(v \mathrm{NH}) ; 3314(v \mathrm{OH}) ;{ }^{1} \mathrm{H}-\mathrm{NMR}(\mathrm{J}, \mathrm{Hz}): 2.44$ $\left(3 \mathrm{H}, \mathrm{s}, \mathrm{Ar}-\mathrm{CH}_{3}\right) ; 4.12\left(2 \mathrm{H}, \mathrm{d}, \mathrm{J}=4.56,-\mathrm{NHCH}_{2}\right) ; 6.85\left(1 \mathrm{H}, \mathrm{t}, \mathrm{J}=4.56,-\mathrm{NHCH}_{2}\right) ; \mathrm{Ar}-\mathrm{H}[7.31(2 \mathrm{H}, \mathrm{d}, \mathrm{H}-1$, $\mathrm{J}=7.80$, p-tolyl); 7.40 (2H, d, H-2, J=7.80, p-tolyl); 7.50 (1H, t, J=8.65, H-8, 2-hydroxy-1-naphthyl); 7.557.64 (2H, m, H-6, 9, 2-hydroxy-1-naphthyl); 7.70 (1H, t, J=8.65, H-7, 2-hydroxy-1-naphthyl); 7.80-8.00 (2H, m, H-10, 11, 2-hydroxy-1-naphthyl)]; $10.21(1 \mathrm{H}, \mathrm{s}, \mathrm{OH}) ;{ }^{13} \mathrm{C} \mathrm{NMR}(\delta, \mathrm{ppm}): 21.07\left(\mathrm{Ar}^{\left.-\mathrm{CH}_{3}\right) ;} 42.87\right.$ $\left(\mathrm{CH}_{2}\right) ; 60.26(\mathrm{C})$; Ar-C [107.99 (C); $115.80(\mathrm{C}) ; 118.90(\mathrm{C}) ; 121.99(2 \mathrm{CH}) ; 123.16(\mathrm{C}) ; 123.77(\mathrm{CH})$; $124.88(\mathrm{CH}) ; 126.50(\mathrm{CH}) ; 127.65(2 \mathrm{CH}) ; 128.19(\mathrm{CH}) ; 128.22(\mathrm{C}) ; 129.00(\mathrm{CH}) ; 129.81(\mathrm{CH}) ; 138.89$ $(\mathrm{CH}) ; 139.55(\mathrm{CH}) ; 149.15(\mathrm{C}) ; 152.00(\mathrm{CH}), 160.26(\mathrm{C})]$; 150.08, $151.32\left(2 \mathrm{C}\right.$, triazole $\left.\mathrm{C}_{5}, \mathrm{C}_{3}\right)$; Anal.calcd. Found, \%: C 72.74; H 5.06; N 14.09. $\mathrm{C}_{24} \mathrm{H}_{20} \mathrm{~N}_{4} \mathrm{O}_{2}$. Calculated, \%: C 72.71; $\mathrm{H} 5.09 ; \mathrm{N}$ 14.13.

\subsection{Antioxidant and Urease Inhibitory Activity Assays}

The DPPH and ABTS radical scavenging activities of the compounds contain triazole moiety were determined using the method described by Williams et al. [26] and Arnao et al. [27], respectively. The reducing power activities of the compounds were examined considering Oyaizu method [28]. Antiurease activities of compounds were assayed according to the procedure of Van Slyke and Archibald [29].

\section{RESULTS AND DISCUSSION}


In current research, the hydrazone compound $\mathbf{2}$ was obtained from the reaction of ethyl pmethylbenzimidate hydrochloride 1, which was synthesized according to reference [25], with furan-2carbohydrazide and the content of the compound is lightened by FT-IR, ${ }^{1} \mathrm{H}-\mathrm{NMR},{ }^{13} \mathrm{C}-\mathrm{NMR}$ and elemental analysis techniques. The compound $\mathbf{3}$ was synthesized by adding hydrazine hydrate to the compound $\mathbf{2}$. The reaction was refluxed for 24 hours using 1-propanol as solvent and the 4- amino-3-furan-2-yl-5-pmethylphenyl-1,2,4-triazole 3 was formed. IR spectrum of compound 3 was determined the $-\mathrm{NH}_{2}$ bands between 3321-3194 $\mathrm{cm}^{-1}$ area. The ${ }^{1} \mathrm{H}-\mathrm{NMR}$ characteristic signal of $\mathbf{3}$ was observed at $\delta 6.28\left(\mathrm{~s}, 2 \mathrm{H}, \mathrm{NH}_{2}\right)$.

The Carbon-NMR signals for the triazole $\mathrm{C}_{3}, \mathrm{C}_{5}$ were observed at $\delta$ 150-155. Synthesis of Schiff bases obtained from the reaction of carbonyl compounds with primary amines takes place in two main steps as separation and addition steps. Firstly, a carbonylamine intermediate compound is formed from the condensation of the carbonyl group with the primary amine, then a Schiff base is obtained from the dehydration of this intermediate compound [30]. Figure 1 shows the formation of synthesized Schiff bases. Synthesized Schiff bases were obtained in high yields by activation of amines and aldehydes in ethanol (\% 80-82). Compounds (4a-c) composed of compound 3 by refluxing with 2-hydroxy-1-benzaldehyde, 1naphthaldehyde and 2-hydroxy-1-naphthaldehyde in acetic acid solvent.

Firstly, we synthesized the ariliden compounds. IR spectra showed the imine peaks of $(\mathbf{4 a}-\mathbf{c})$ in the 1530$1640 \mathrm{~cm}^{-1}$ area. When the Proton-NMR spectra of Schiff bases are examined, the most important peak to be considered is the peak belonging to the azomethine proton, which is the characteristic peak of this type of compounds. The proton bound to the azomethine group is generally resonance in the range of $\delta=8-9$ ppm. When the ${ }^{1} \mathrm{H}-\mathrm{NMR}$ spectrum of the obtained Schiff base compounds was examined, the peaks of azomethine hydrogens were observed as $\delta=8.69-9.22 \mathrm{ppm}$ and singlet as expected in all three compounds. Peaks of imine carbons are seen in the ${ }^{13} \mathrm{C}$-NMR spectrum between $\delta=164.6-171.5 \mathrm{ppm}$. Imine peak emerged as a singlet. It was observed that the NMR results supported the formation of the compound and were consistent with the literature [31]. Reduction in reduced products $(\mathbf{5 a}-\mathbf{c})$ was took place only the nonring $\mathrm{C}=\mathrm{N}$ bond of the ariliden compounds (4a-c) (Figure 1). These degradation reactions were happening in fairly moderate conditions.

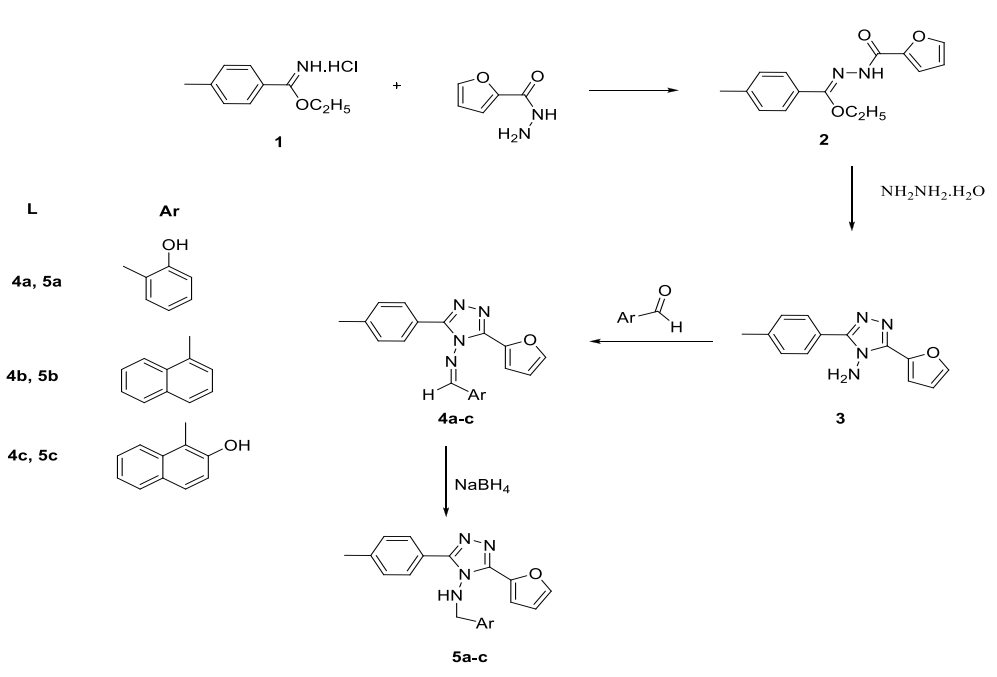

Figure 1. Synthesized tigands

Reduction products $(\mathbf{5 a}-\mathbf{c})$ represent IR peaks between $3250-3320 \mathrm{~cm}^{-1}(\mathrm{vNH})$. The proton-NMR characteristic signals of $(\mathbf{5 a}-\mathbf{c})$ were observed as a triplet at $\delta 6.74-6.85(\mathrm{t}, 1 \mathrm{H}, \mathrm{NH})$ and as a doublet at $\delta$ 3.92-4.29 (d, $\left.2 \mathrm{H}, \mathrm{CH}_{2}\right)$. The $-\mathrm{CH}_{2}-$ signals of compounds $(\mathbf{5 a}-\mathbf{c})$ were observed between $\delta 43-52$. These results were consistent with the literature [31]. The reducing capacity of a compound may serve as a significant indicator for its potential antioxidant activity [32]. The reducing power activities of the compounds contain azomethine group were viewed at various concentrations $(50-200 \mu \mathrm{g} / \mathrm{mL})$, and findings were compared with BHT (Table 1).

Table 1. The reducing power antioxidant activity of new ligands (2, 3, 4a-c, 5a-c) 


\begin{tabular}{ccccc}
\hline Comp. & $\begin{array}{c}\text { Ligands } \\
\text { Concentrati } \\
\text { on } \\
\boldsymbol{\mu g} / \mathbf{m L})\end{array}$ & $\begin{array}{c}\text { Reducing } \\
\text { Power } \\
\text { Absorbance* }\end{array}$ & Comp. & $\begin{array}{c}\text { Reducing } \\
\text { Power } \\
\text { Absorbance* }\end{array}$ \\
\hline \multirow{2}{*}{2} & 50 & $0.018 \pm 0.004$ & & $0.037 \pm 0.003$ \\
& 100 & $0.032 \pm 0.004$ & $5 \mathrm{a}$ & $0.054 \pm 0.007$ \\
& 150 & $0.046 \pm 0.007$ & & $0.072 \pm 0.009$ \\
& 200 & $0.059 \pm 0.001$ & & $0.089 \pm 0.006$ \\
3 & 50 & $0.193 \pm 0.026$ & & $0.137 \pm 0.002$ \\
& 100 & $0.276 \pm 0.012$ & $5 \mathrm{~b}$ & $0.146 \pm 0.004$ \\
& 150 & $0.311 \pm 0.014$ & & $0.161 \pm 0.009$ \\
$4 \mathrm{a}$ & 200 & $0.469 \pm 0.024$ & & $0.192 \pm 0.004$ \\
& 50 & $0.075 \pm 0.004$ & & $0.068 \pm 0.004$ \\
& 100 & $0.088 \pm 0.009$ & $5 \mathrm{c}$ & $0.082 \pm 0.006$ \\
$4 \mathrm{~b}$ & 150 & $0.112 \pm 0.013$ & & $0.113 \pm 0.016$ \\
& 200 & $0.155 \pm 0.009$ & & $0.174 \pm 0.012$ \\
& 50 & $0.044 \pm 0.008$ & & $0.162 \pm 0.012$ \\
& 100 & $0.079 \pm 0.005$ & $\mathrm{BHT}$ & $0.205 \pm 0.018$ \\
$4 \mathrm{c}$ & 150 & $0.121 \pm 0.001$ & & $0.274 \pm 0.021$ \\
& 200 & $0.137 \pm 0.008$ & & $0.311 \pm 0.025$ \\
& 50 & $0.067 \pm 0.002$ & & \\
\hline
\end{tabular}

*Values were the means of three replicates \pm Standard deviation (SD).

In this study, the reducing power capacity of newly synthesized compounds rised with increasing concentration of samples. Compounds $4 \mathrm{~b}$ and $4 \mathrm{c}$ showed similar results. In the same concentrations, compounds 3 and 2 showed the highest $(0.469 \pm 0.024)$ and lowest $(0.059 \pm 0.001)$ activities, respectively. Compound 3 showed higher activity than BHT at all concentration.

Gumrukcuoglu et al. (2013) have synthesized new 1,4-butylene bridged bis-1,2,4-triazole derivatives and their reducing power antioxidant capacity absorbance values found between $0.121-0.143$ [33]. In this study, the reducing power absorbance values of triazole derivatives are between 0.059-0.469 in the range of.

Table 2. The antioxidant and antiurease activities of new ligands and standards (2, 3, 4a-c, 5a-c)

\begin{tabular}{|c|c|c|c|}
\hline Compounds & DPPH SC $50(\mu \mathrm{M}) *$ & $\operatorname{ABTS~SC} 50(\mu M) *$ & Antiurease $\left.\mathrm{IC}_{50} \boldsymbol{\mu M}\right)$ * \\
\hline 2 & $12809.28 \pm 2502.59$ & $2877.73 \pm 287.07$ & $0.99 \pm 0.013$ \\
\hline 3 & $5071.17 \pm 382.07$ & $1119.6 \pm 95.72$ & $0.12 \pm 0.009$ \\
\hline $4 a$ & $9737.89 \pm 699.02$ & $1874.72 \pm 127.68$ & $0.15 \pm 0.004$ \\
\hline $4 b$ & $5334.98 \pm 207.39$ & $1628.28 \pm 25.73$ & $0.14 \pm 0.001$ \\
\hline $4 c$ & $11749.17 \pm 431.96$ & $1299.17 \pm 108.79$ & $0.14 \pm 0.013$ \\
\hline $5 \mathrm{a}$ & $5324.36 \pm 582.73$ & $2421.09 \pm 398.41$ & $0.16 \pm 0.011$ \\
\hline $5 b$ & $4542.71 \pm 197.44$ & $806.02 \pm 1.43$ & $0.22 \pm 0.027$ \\
\hline $5 c$ & $6512.24 \pm 981.80$ & $1063.63 \pm 43.41$ & $0.29 \pm 0.039$ \\
\hline Trolox & $132.03 \pm 9.75$ & $214.55 \pm 24.56$ & - \\
\hline Thiourea & - & - & $0.11 \pm 0.004$ \\
\hline
\end{tabular}

*Values were the means of three replicates \pm Standard deviation (SD).

DPPH and ABTS radical scavenging assays have been used to evaluate the antioxidant activity of compounds due to the simple, rapid, and reproducible procedures of compounds. The DPPH and ABTS radical scavenging activities of new ligands are presented in Table 2. As standard antioxidant, Trolox (6hydroxy-2,5,7,8-tetramethylchroman-2-carboxylic acid) was used. Their comparable scavenging efficiency were stated in SC50 (50\% of radicals in the discarded activity) value. Compound 5b (SC50 = 4542.71 \pm 
$197.44 \mu \mathrm{M}$ for DPPH; SC50 $=806.02 \pm 1.43 \mu \mathrm{M}$ for ABTS) and $2(\mathrm{SC} 50=12809.28 \pm 2502.59 \mu \mathrm{M}$ for $\mathrm{DPPH}$; SC50 $=2877.73 \pm 287.07 \mu \mathrm{M}$ for ABTS) exhibited the highest and the lowest scavenging activities among all the tested compounds, respectively. Scavenging activity values of the compounds were lower than Trolox. DPPH is a free radical compound that has been widely used to determine the free radical scavenging ability of various samples. DPPH decreases significantly upon exposure to proton radical scavenger [34]. Bilgin Sokmen et al. (2015) have synthesized newly 1,2,4-bis triazole derivatives and their DPPH SC50 values found between 4376.08- 13337.95 $\mu \mathrm{M}$ [35]. Bilgin Sokmen et al. (2013) have synthesized some new methoxy substitued bis-1,2,4-triazole derivatives and their ABTS radical scavenging activities values (SC50) determined between 3274.88- 8734.22 $\mu \mathrm{g} / \mathrm{mL}$ [36].

As seen in Table 2, the original compounds showed moderate urease inhibitory activity. Inhibition values were increased with increasing sample concentration. Higher enzyme inhibitory activity shows low IC50 value. The highest and lowest enzyme inhibition activities were found at compounds 3 (IC50 $=0.12 \pm 0.009$ $\mu \mathrm{M})$ and 2 (IC50 $=0.99 \pm 0.013 \mu \mathrm{M})$, respectively. Triazoles have been regarded as structural type inhibitors of urease. 1,2,3 - Triazole and 1,3,4-thiadiazole derivatives were synthesized by Khan et al. showed potent urease inhibitor activity between IC50=45.60- $459.56 \mu \mathrm{M}$ [37]. Gumrukcuoglu et al. reported that of the antiurease IC50 values of the 1,2,4-triazole derivatives were between $2.44-2.62 \mu \mathrm{M}$ [33].

\section{CONCLUSION}

In current study, the results exhibit that the obtained new ligands containing azomethine group had antioxidant and an effective antiurease activities. Consequently, these substances are considered to be antioxidant agents in technology such as medicine uses, food additives, industrial uses as stabilizers in fuels and lubricants to prevent oxidation.

Recently, many scientist are showing a great interest in the synthesis and physico-chemical properties of transition metal complexes with substituted 1,2,4-triazoles. Triazoles and their derivatives have been evidenced to be effective bactericides, pesticides, fungicides and insecticides [38, 39]. Many Schiff bases obtained from either heterocyclic amines or aldehydes possess excellent ability to synthesis transition metals complexes [40].

Thus the main idea of our present research work is to synthesize triazole derived Schiff bases as well as to synthesis variety of metal complexes with various transition metal ion.

\section{CONFLICTS OF INTEREST}

No conflict of interest was declared by the authors.

\section{REFERENCES}

[1] Carter, M.J., Rillema, D.P., Basolo, F.J., "Oxygen carrier and redox properties of some neutral cobalt chelates. Axial and in-plane ligand effects", Am. Chem. Soc., 96: 392-400, (1974).

[2] Asada, H., Hayashi, K., Negoro, S., Fujiwara, M., Matsushita, T., "Preparation and structures of tri nuclear manganese (II) complexes with N-2-pyridiylmethylidene-2-hydroxy-5-substitutedphenylamine", Inorg. Chem. Commun., 6: 193-196, (2003).

[3] Saghatforoush, L., Aminkhani, A., Ershad, S., Karimnezhad, G., Ghammamy, S., Kabiri, R., "Preparation of Zinc (II) and Cadmium (II) Complexes of the Tetradentate Schiff Base Ligand 2-((E)(2-(2-(pyridine-2-yl)-ethylthio)ethylimino)methyl)-4-bromophenol (PytBrsalH)", Molecules, 13: 804$811,(2008)$.

[4] Li, S., Chen, S., Ma, H., Yu, R., Liu, D.,"Investigation on some Schiff bases as $\mathrm{HCl}$ corrosioninhibitors for copper", Corros. Sci., 41: 1273-1287, (1999). 
[5] Khalifa, K.M., Maihub, A.A., El-Ajaily, M.M., Mobain, S.A., "Coordination Trends of 6-Amino-4hydroxy-2-mercaptopyrimidine Towards $\mathrm{Co}$ (II), Ni (II) and Cu (II) Ions", J. Chem. Soc. Pak., 32: 650$653,(2010)$.

[6] Unaleroglu, C., Temelli, B., Hokelek, T., "Conformational and structural analysis of N-N'-bis(4methoxybenzylidene)ethylenediamine”, J. Mol. Struct., 570: 91-95, (2001).

[7] Barba, V., Santillan, R., Farfan, N., "Dimeric Boronates Derived from the Reaction of Schiff Bases and Boronic Acids", J. Braz. Chem. Soc., 16: 449-455, (2005).

[8] Chen, W., Ou, W., Wang, L., Hao, Y., Cheng, J., Li, J., Liu, Y.N., "Synthesis and biological evaluation of hydroxyl-substituted Schiff-bases containing ferrocenyl moieties", Dalton Trans. 42: 15678-15686, (2013).

[9] Cheng, L., Tang, -X., Luo, X., Jin, -L., Dai, F., Yang, J., Y.-P. Qian, Li, X.-Z., Zhou, B., “Antioxidant and antiproliferative activities of hydroxyl-substituted Schiff bases", Bioorg. Med. Chem. Lett., 20: 2417-2420, (2010).

[10] Norman, D.W., Edwards, J.P., Vogels, C.M., Decken, A.S., Westcott, A., "Synthesis and reactivity of novel Schiff bases containing boronate esters", Can. J. Chem., 80: 31-40, (2002).

[11] Singh, R.V., Biyala, M.K., "Structural studies with antimicrobial and antifertility activity of a monofunctional bidentate ligand with its boron(III), palladium(II), and platinum(II) complexes", Phosphorus Sulfur Silicon Relat. Elem., 181: 1477-1491, (2006).

[12] Hou, A., Zhang, C., Wang, Y., "Preparation and UV-protective properties of functional cellulose fabrics based on reactive azobenzene Schiff base derivative", Carbohydr. Polym., 87: 284-288, (2012).

[13] Farag, A.M., Siang, T.G., Osman, H., Eltayeb, N.E., Khadeer Ahamed, M.B., Abdul Majid, A.M.S., Balal, I.A.A., "Synthesis, characterizationand in vitro cytotoxic properties of some novel Schiff base metal complexes in HepG2 cells", World Appl. Sci. J., 11: 1196-1209, (2010).

[14] Sarı, N., Yüzüak, N., "Synthesis and characterization of novel polymeric-schiff bases and their complexes", J. Inorg. Organomet. Polym. Mater., 16: 259-269, (2006).

[15] Rice-Evans, C.A., Diplock, A.T., Symons, M.C.R., Techniques in Free Radical Research 1st ed., Elsevier, Amsterdam, (1991).

[16] Griendling, K.K., FitzGerald, G.A., "Oxidative stress and cardiovascular injury: Part I: basic mechanisms and in vivo monitoring of ROS", Circulation, 108: 1912-1916, (2003).

[17] Reuter, S., Gupta, C.S., Chaturvedi, M.M., Aggarwal, B.B. "Oxidative stress, inflammation, and cancer: How are they linked?”, Free Radic. Biol. Med., 49: 1603-1616, (2010).

[18] Nihro, S.Y., Ueda, T., Miki, H., Matsomota, H., Satoh, T., "Protective effects of hydroxychalcones on free radical-induced cell damage", Biol. Pharm. Bull., 17: 251-256, (1994).

[19] Zerner, B., "Recent advances in the chemistry of an old enzyme, urease", Bioorg. Chem., 19: 116-131, (1991).

[20] Krajewska, B., Van-Eldik, R., Brindell, M., "Temperature- and pressure-dependent stopped-flow kinetic studies of jack bean urease", Implications for the catalytic mechanism, J. Biol. Inorg. Chem., 17: 1123-1134, (2012). 
[21] Mobley, H.L.T., Island, M.D., Hausinger, R.P., "Molecular biology of microbial ureases”, Microbiol. Rev., 59: 451-480, (1995).

[21] Mobley, H.L.T., Island, M.D., Hausinger, R.P., "Molecular biology of microbial ureases", Microbiol. Rev., 59: 451-480, (1995).

[22] Warren, J.R. Marshall, B., "Unidentified curved bacilli on gastric epithelium in active chronic gastritis", Lancet., 1: 1273-1275, (1983).

[23] Maroney, M., Ciurli, J. S., "Nonredox Nickel Enzymes”, Chem. Rev., 114: 4206-4228, (2014).

[24] Mazzei, L., Cianci, M., Benini, S., Bertini, L., Musiani, F. Ciurli, S., "Inactivation of urease by 1, 4benzoquinone: chemistry at the protein surface", J. Inorg. Biochem., 154: 42-49, (2016).

[25] Gumrukcuoglu, N., Serdar, M., Celik, E., Sevim, A., Demirbas, N., "Synthesis and Antimicrobial Activities of Some New 1, 2, 4-Triazole Derivatives", Turk. J. Chem., 31: 335-348, (2007).

[26] Brand-Williams, W., Cuvelier, M.W. and Berset, C., "Use of a free radical method to evaluate antioxidant activity", LWT-Food Sci. Technol., 28: 25-30, (1995).

[27] Arnao, M.B., Cano, A., Acosta, M., "The hydrophilic and lipophilic contribution to total antioxidant activity", Food Chem., 73: 239-244, (2001).

[28] Oyaizu, M., “Studies on Products of Browning Reaction”, Japan. J. Nutr., 44: 307-315, (1986).

[29] Van Slyke, D.D. and Archibald, R.M., "Manometric, Titrimetric and Colorimetric Methods for Measurement of Urease Activity", BJ. Biol. Chem., 154: 623-642, (1944).

[30] Nelson, S.M., Knox, C.V., Cann Drew, M.Mc., "Metal-ion-controlled transamination in the synthesis of macrocyclic Schiff-base ligands. Part I. Reactions of 2, 6-diacetylpyridine and dicarbonyl compounds with 3, 6-dioxaoctane-1,8-diamine", J. Chem. Soc. Dalton Trans., 8: 1669-1677, (1981).

[31] Gumrukcuoglu, N., Ugras, S., Ugras, H.I., Cakir, U., "Synthesis, extraction and antibacterial studies of some new bis-1,2,4-triazole derivatives part II", J. Incl. Phenom. Macrocycl. Chem., 73: 359-367, (2012).

[32] Meir, S., Kanner, J., Akiri, B., Hadas, S.P., "Determinating and involvement of aqueus reducing compounds in oxidative defense systems of various senescing leaves", J. Agric. Food Chem., 43: 18131819, (1995).

[33] Gumrukcuoglu, N., Bilgin Sokmen, B., Ugras, S., Ugras, H. I., Yanardag, R. "Synthesis, antibacterial, antielastase, antiurease and antioxidant activities of new 1,4-butylene bridged bis-1,2,4-triazole derivatives", J. Enzyme Inh. Med. Chem., 28: 89-94, (2013).

[34] Yamaguchi T, Takamura H, Matoba T, Terao J. HPLC method for evaluation of the free radicalscavenging activity of foods by using 1,1-diphenyl-2-picrylhydrazyl. Biosci. Biotechnol. Biochem., 62: 1201-1204, (1998).

[35] Bilgin Sokmen, B., Gumrukcuoglu, N., Ugras, S., Sahin, H., Sagkal, Y., Ugras, H.I., "Synthesis, Antibacterial, Antiurease, and Antioxidant Activities of Some New 1,2,4-Triazole Schiff Base and Amine Derivatives", Appl. Biochem. Biotech, 175: 705-714, (2015).

[36] Bilgin Sokmen, B., Gumrukcuoglu, N., Ugras, S., Ugras, H. I., Yanardag, R. "Synthesis, antibacterial, antielastase, antiurease and antioxidant activities of new methoxy substitued bis-1,2,4-triazole derivatives", J. Enzyme Inh. Med. Chem., 28: 72-77, (2013). 
[37] Khan, I., Ali, S., Hameed, S., Rama, N.H., Hussain, M.T., Wadood, A., et al. "Synthesis, antioxidant activities and urease inhibition of some new 1,2,4-triazole and 1,3,4-thiadiazole derivatives", Eur. J. Med. Chem., 45: 5200-5207, (2010).

[38] Pombeiro, A. J. L., Kukushkin, V. Yu., “Comprehensive Coordination Chemistry II”, J.A. McCleverty and T.J. Meyer, Eds.; Elsevier-PergamonPress, Oxford, New York, 1: 585 (2003).

[39] Kukushkin, V. Yu., Pombeiro, A. J. L., Additions to Metal-Activated Organonitriles Chem. Rev., 102: 1771-1802, (2002).

[40] Steed, J. W., Atwood, J. L., "Definition and Emergence of Supramolecular Chemistry Adapted in part from Supramolecular Chem", Wiley: Chichester, 2nd Ed., 2009. 